Proceedings of the ASME Turbo Expo 2016:

Turbomachinery Technical Conference \& Exposition

June 13-17, 2016, Seoul, South Korea

GT2016-56700

\title{
DNS OF LOW-PRESSURE TURBINE CASCADE FLOWS WITH ELEVATED INFLOW TURBULENCE USING A DISCONTINUOUS-GALERKIN SPECTRAL-ELEMENT METHOD
}

\author{
Anirban Garai \\ Oak Ridge Associated Universities \\ NASA Ames Research Center \\ Moffett Field, CA, USA
}

\author{
Laslo T. Diosady \\ Science and Technology Corporation \\ NASA Ames Research Center \\ Moffett Field, CA, USA
}

\author{
Scott M. Murman \\ Nateri K. Madavan \\ NASA Ames Research Center \\ Moffett Field, CA, USA
}

\begin{abstract}
Recent progress towards developing a new computational capability for accurate and efficient high-fidelity direct numerical simulation (DNS) and large-eddy simulation (LES) of turbomachinery is described. This capability is based on an entropystable Discontinuous-Galerkin spectral-element approach that extends to arbitrarily high orders of spatial and temporal accuracy, and is implemented in a computationally efficient manner on a modern high performance computer architecture. An inflow turbulence generation procedure based on a linear forcing approach has been incorporated in this framework and DNS conducted to study the effect of inflow turbulence on the suctionside separation bubble in low-pressure turbine (LPT) cascades. The T106 series of airfoil cascades in both lightly (T106A) and highly loaded (T106C) configurations at exit isentropic Reynolds numbers of 60,000 and 80,000, respectively, are considered. The numerical simulations are performed using 8th-order accurate spatial and 4th-order accurate temporal discretization. The changes in separation bubble topology due to elevated inflow turbulence is captured by the present method and the physical mechanisms leading to the changes are explained. The present results are in good agreement with prior numerical simulations but some expected discrepancies with the experimental data for the T106C case are noted and discussed.
\end{abstract}

\section{NOMENCLATURE}

$\boldsymbol{A}_{0} \quad$ Jacobian of entropy to conservative variables
$\boldsymbol{A}_{i} \quad$ Flux Jacobian of entropy variables

$\boldsymbol{K}$ Diffusion tensor

$B \quad$ Linear forcing parameter

$C \quad$ Axial chord length

$C_{f} \quad$ Wall shear stress coefficient

$C_{p} \quad$ Airfoil surface pressure

E Total energy

$M a_{i s} \quad$ Isentropic Mach number

$P D$ Pressure dilatation

PML Perfectly Matched Layer

$R \quad$ Gas constant

Re Reynolds number

$R e_{\lambda} \quad$ Taylor microscale Reynolds number

$T$ Temperature

$\mathrm{Tu} \quad$ Turbulence intensity

$f_{e} \quad$ Source term for total energy equation

$k \quad$ Turbulent kinetic energy

$\ell \quad$ Integral length scale

$p \quad$ Pressure

$s \quad$ Entropy

$t$ Time

$u \quad$ Velocity

$u_{t} \quad$ Turbulent velocity

$v \quad$ Entropy variables

$\boldsymbol{w}$ Basis functions

$x \quad$ Streamwise coordinate direction

$y \quad$ Pitchwise coordinate direction

$z \quad$ Spanwise coordinate direction 


$\begin{array}{ll}\varepsilon & \text { Dissipation } \\ \eta & \text { Wall-normal direction } \\ \gamma & \text { Specific heat ratio } \\ \kappa & \text { Thermal conductivity } \\ \omega_{u} & \text { Wake deficit } \\ \rho & \text { Density } \\ \tau & \text { Shear stress } \\ \xi & \text { Tangential direction } \\ \text { Subscripts } \\ 1 & \text { Inflow } \\ 2 & \text { Outflow } \\ t & \text { Total condition }\end{array}$

\section{INTRODUCTION}

Design trends in modern high bypass ratio gas turbine engines require the low-pressure turbine (LPT) stages to drive increasingly larger fans without compromising efficiency. In addition, in order to reduce weight and costs, LPT stages are being designed with fewer highly loaded airfoils in each row. The resulting increased airfoil loadings are characterized by low suction-side peak pressures, followed by a region of strong diffusion in the aft portion of the airfoils where the adverse pressure gradient results in boundary layer separation and a loss in efficiency. In modern gas turbines, typically about a $2 \%$ loss in LPT efficiency occurs between take-off (Reynolds number around $5 \times 10^{5}$ ) and high altitude cruise (Reynolds number around $5 \times 10^{4}$ ) conditions. Suction-side boundary layer separation is a major source of loss of efficiency in LPT airfoils. The boundary layer on the suction side initially remains laminar due to the high acceleration in the fore region and generally separates just after the peak. This separated boundary layer may become transitional due to the lower Reynolds number at high altitude cruise. Reattachment in the aft region of the airfoil may occur if the transition occurs early enough in the separated shear layer [ 1 , and the references cited therein].

The transition from laminar to turbulent flow typically occurs through three distinct modes: natural transition by the process of amplification of Tollmien-Schlichting waves, "bypass" transition in the presence of high inflow turbulence, or separation-induced transition in the shear layer. Separationinduced transition in the suction-side separation bubble is quite common in low Reynolds number LPT applications. These separation bubbles are classified as either long or short based on their effect on the airfoil pressure distribution. Long separation bubbles modify the overall pressure distribution, causing high losses and lower exit flow angles, while short bubbles modify the pressure distribution only locally compared to the designed pressure distribution when the flow is attached [2]. Short bubble topologies are obviously preferable in order to avoid performance detriments. The topologies of the separation bubbles in LPT airfoils can be modified by the Reynolds number, flow angle, and inflow turbulence levels [1].

Current Reynolds averaged Navier-Stokes (RANS) -based methods for turbomachinery flow prediction have proven extremely useful, despite their inherent limitations in modeling transitional and turbulent flows in complex configurations. The rapid expansion of computing capability in recent years has led to the consideration of high-fidelity simulation techniques, such as DNS and LES. These techniques offer the promise of more accurate and better-resolved simulations that can shed more light on the complicated flow phenomena, and lead to improved turbomachinery designs. Incompressible DNS have been reported [3, and the references cited therein] to study the effect of incoming wakes on transition. Compressible DNS of effects such as flow transition and rotor-stator interaction in turbomachinery have been performed using high-order upwind schemes [4-7] and 4thorder compact finite-difference schemes [8-10]. These studies were generally performed on idealized mid-span configurations and at low Reynolds numbers to keep the computational costs reasonable.

Discontinuous-Galerkin (DG) schemes for simulations of flows in complex geometries have recently been gaining attention due to their many attractive features, including their extensibility to arbitrarily high-order schemes on compact numerical stencils, applicability to different element types and mesh topologies, and computational efficiency. The use of DG methods in turbomachinery flow simulations is relatively new, and both RANSbased turbomachinery simulations [11-14] and DNS simulations [15-18] using DG methods have been reported in the literature in recent years.

Since gas turbine engines typically operate in regimes with elevated turbulence intensity levels, accurate simulations of turbomachinery flows require the development of numerical approaches to incorporate these effects. A variety of approaches have been reported in the literature, ranging from purely numerical methods such as blowing-suction [4] and synthetic turbulence generation $[9,10]$ that are somewhat empirical and require calibration, to stochastic and deterministic forcing methods that generate homogeneous isotropic turbulence (HIT) by attempting to represent the physical processes more accurately (cf. [19]). One such method that is based on the latter approach and perturbs the low turbulence wavenumbers has been applied to LPT simulations by Medic and Sharma [20]. In the current work, a linear forcing method developed by Lundgren [21] is adapted for the DG framework and used to generate HIT upstream of the LPT cascades to match the experimental conditions.

In the present paper, an inflow turbulence generation procedure for turbomachinery applications based on a linear forcing approach is developed in the DG framework. DNS studies are then conducted to study the effect of inflow turbulence on the suction-side separation bubble in LPT cascades. The T106 series airfoil cascades in both lightly loaded (T106A) and highly 
loaded (T106C) configurations at exit isentropic Reynolds numbers of 60,000 and 80,000, respectively, are considered. By studying the same airfoil section under different loading, the importance (or lack thereof) of including inflow turbulence is examined across trends. The numerical simulations are performed using 8th-order accurate spatial and 4th-order accurate temporal discretization. The main objective of the paper is to study the effect of inflow turbulence on the suction-side separation bubble topology in these LPT cascades.

The paper is organized as follows. The inflow turbulence generation and the DG numerical method are first described. The ability of the inflow turbulence generation method to achieve HIT with specified target conditions is then demonstrated, followed by detailed simulation results and conclusions for the T106A and T106C cascade configurations.

\section{INFLOW TURBULENCE GENERATION METHOD}

In order to predict turbomachinery flows with elevated inflow turbulence, we seek an approach that generates HIT with the correct $R e_{\lambda}$ in the computational region upstream of the airfoil cascade and accurately reproduces the test conditions in the experiment. Physics-based methods for generating HIT typically involve either perturbing the low wavenumber turbulence through a stochastic process $[19,22]$, or injecting energy at low wavenumbers at a constant rate $[23,24]$ or forcing low wavenumber at constant energy level [25,26]. These methods are developed in wavenumber space and are thus global in nature. Due to the local nature of the DG formulation, local methods that are developed in physical space are preferred. Lundgren proposed a "linear forcing method" for incompressible flows, that is applied in physical space and excites all wavenumbers [21]. This method was subsequently extended to improve the convergence to a stationary state [27], and for compressible flows [28, 29]. In the present work, we develop a compressible formulation of this method that can be incorporated in the DG finite-element framework and discuss its required inputs and stability properties.

The turbulent kinetic energy equation for a compressible fluid is given by:

$$
\begin{array}{r}
\frac{\partial\{\rho k\}}{\partial t}+\frac{\partial<\rho>\left\{u_{k}\right\}\{k\}}{\partial x_{k}}=\left[-<\rho>\left\{u_{k}^{\prime \prime} u_{k}^{\prime \prime}\right\} \frac{\partial\left\{u_{i}\right\}}{\partial x_{k}}\right] \\
+\left[-\frac{\partial}{\partial x_{k}}\left(<\rho>\left\{u_{k}^{\prime \prime} k^{\prime \prime}\right\}+<p^{\prime} u_{k}^{\prime}>+<\tau_{i k}^{\prime} u_{i}^{\prime}>\right)\right] \\
+\left[-<\tau_{i k}^{\prime} \frac{\partial u_{i}^{\prime}}{\partial x_{k}}>+<u_{i}^{\prime \prime}>\frac{\partial<\tau_{i k}>}{\partial x_{k}}\right] \\
+\left[-<u_{k}^{\prime \prime}>\frac{\partial<p>}{\partial x_{k}}+<p^{\prime} \frac{\partial u_{k}^{\prime}}{\partial x_{k}}>\right]
\end{array}
$$

where $<>,\{\}$ are the ensemble- and Favre-averaged quantities respectively, and ', " are the fluctuations from these respective quantities. The first, second, third, and fourth terms on the right hand side of Eqn. (1) are the energy production, diffusion, dissipation, and pressure dilatation $(P D)$ terms, respectively. In the case of homogeneous turbulence, the production and diffusion terms are zero, and turbulence dissipation and $P D$ is balanced by the decay of turbulent kinetic energy. Forcing, typically in the form of volumetric source terms, must be added in order to sustain homogeneous turbulence at a given level. Since the turbulence production term in Eqn. (1) is proportional to the fluctuations, one choice is to use source terms that are proportional to the fluctuations for homogeneous turbulence [21, 27-29].

For homogeneous compressible flow, and assuming zero mean flow (without any loss of generality), the Navier-Stokes equations with a compressible extension of Lundgren's linear forcing source terms are:

$$
\begin{gathered}
\frac{\partial \rho}{\partial t}+\frac{\partial \rho u_{j}}{\partial x_{j}}=0 \\
\frac{\partial \rho u_{i}}{\partial t}+\frac{\partial \rho u_{i} u_{j}}{\partial x_{j}}=-\frac{\partial p}{\partial x_{i}}+\frac{\partial \tau_{i j}}{\partial x_{j}}+B \rho u_{i} \\
\frac{\partial \rho E}{\partial t}+\frac{\partial \rho E u_{j}}{\partial x_{j}}=-\frac{\partial u_{j} p}{\partial x_{j}}+\frac{\partial u_{i} \tau_{i j}}{\partial x_{j}}-\frac{\partial q_{j}}{\partial x_{j}}+B \rho u_{j} u_{j}+f_{e} .
\end{gathered}
$$

Note that all turbulence modes in the solution are perturbed by adding $B \rho u_{i}$ as the source term.

For homogeneous flow, the volume-averaged energy Eqn. (2c) becomes

$$
\frac{\partial<\rho E>}{\partial t}=<B \rho u_{j} u_{j}>+<f_{e}>
$$

For the total energy to attain a statistically stationary state, one requires $\left\langle f_{e}\right\rangle=-\left\langle B \rho u_{j} u_{j}\right\rangle$ in Eqn. (3). Hence, we set $f_{e}=-B \rho u_{j} u_{j}$ in the equation for total energy, Eqn. (2c) [29].

The volume-averaged turbulent kinetic energy Eqn. (1) with the source term for homogenous flow can now be written as:

$$
\frac{\partial\{\rho k\}}{\partial t}=-\varepsilon-P D+B<\rho u_{j} u_{j}>
$$

Setting $B=\frac{\varepsilon+P D}{<\rho u_{j} u_{j}>}=\frac{\varepsilon+P D}{2\{\rho k\}}$ will result in the turbulent kinetic energy attaining statistical stationarity [21,27-29]. Thus, in order to simulate stationary homogeneous turbulence, one needs to specify $\varepsilon, P D$ and $\{\rho k\}$ at the stationary state. 
For low turbulent Mach numbers, the proportionality constant $B$ can be approximated as $\frac{\varepsilon}{<\rho u_{j} u_{j}>}=\frac{\varepsilon}{2\{\rho k\}}$, since the ratio between $P D$ and $\varepsilon$ scales with the square of turbulent Mach number for homogeneous turbulence [28]. However, large oscillations in the turbulence statistics were noted $[21,28]$ when $B$ was held constant during the simulations (i.e., to specify $\varepsilon$, and $\{\rho k\}$ at the stationary state). Hence, a method for dynamically adjusting $B$ (i.e., to specify $\varepsilon$ at the stationary state, and compute $\{\rho k\}$ instantaneously) was proposed [27] to drive the velocity field towards the desired turbulent state in a more constrained fashion.

In incompressible HIT, the turbulence dissipation, $\varepsilon$, can be estimated as $\frac{u_{t}^{3}}{\ell}$, where $u_{t}$ and $\ell$ are the turbulent velocity and turbulent integral length scale, respectively. The $\varepsilon$ can be approximated as $\frac{u_{t}^{3}}{\ell}$ (since the ratio of the dilatational dissipation to the incompressible dissipation scales as the fourth power of turbulent Mach number [28]) for low turbulent Mach numbers. For linear forcing, the resulting integral length scale is estimated to be approximately $20 \%$ of the computational domain [27]. Thus, by specifying only the turbulent Mach number and choosing a specific domain size, a statistically stationary HIT for low turbulent Mach number flows can be achieved. In the present study, we consider low turbulent Mach number simulations and compute $B$ dynamically.

Eqn. (2) can be rewritten using entropy variables as:

$$
\boldsymbol{A}_{0} \boldsymbol{v}_{, t}+\boldsymbol{A}_{i} \boldsymbol{v}_{, i}=\left(\boldsymbol{K}_{i j} \boldsymbol{v}_{, j}\right)_{, j}+\mathscr{F}
$$

where $v=\left\{\frac{s}{\gamma-1}+\frac{\gamma+1}{\gamma-1}-\frac{\rho E}{p}, \frac{\rho u_{j}}{p},-\frac{\rho}{p}\right\}$, and the source term $\mathscr{F}=\left\{0, B \rho u_{1}, B \rho u_{2}, B \rho u_{3}, 0\right\} ; \boldsymbol{A}_{0}$ and $\boldsymbol{A}_{i}$ are symmetric, and $\boldsymbol{K}_{i j}$ is symmetric and positive-semidefinite. The global entropy conservation equation is derived by taking the dot product of $\boldsymbol{v}$ with Eqn. (5) and integrating over the domain [30,31]. The global entropy evolution is given by:

$$
\int_{\Omega}\left(\frac{\rho s}{\gamma-1}\right)_{, t}+\int_{\Omega} \boldsymbol{v}_{, i} \boldsymbol{K}_{i j} \boldsymbol{v}_{, j}=\int_{\Omega} B \frac{\rho u_{k} u_{k}}{p / \rho}
$$

Ensemble averaging we recover:

$$
<\boldsymbol{v}_{, i} \boldsymbol{K}_{i j} \boldsymbol{v}_{, j}>=<B \frac{\rho u_{k} u_{k}}{p / \rho}>
$$

Thus, the flow will reach a stationary state where the entropy generated through viscous dissipation is exactly balanced by the forcing. Our entropy stable numerical formulation recovers the discrete analog of Eq. (7), ensuring the numerical stability of the scheme.

In turbomachinery simulations, it is often necessary to generate HIT in a computational box that is anisotropic due to geometry constraints. Since all the modes of the momentum Eqn [2b] are being perturbed in this linear forcing method, it is inconvenient to achieve the desired level of turbulence at the stationary state in an anisotropic domain. One way to overcome this is by perturbing only selected modes, or by generating the turbulence in multiple unit (isotropic) cubes that are then stacked on one another. The former procedure requires global operations and is thus less attractive for DG applications. In the present approach, HIT is generated in multiple unit cubes which are then coupled into the main computational domain through a DG boundary. Also, the forcing term in Eqn. [2b] is modified as $B\left(\rho u_{i}-<\rho u_{i}>\right)$ for each individual block in order to ensure that the zero-th mode is not perturbed.

\section{DG METHOD}

A space-time DG discretization method is used to solve the conservative form of the compressible Navier-Stokes equation in entropy variables in conjunction with the linear forcing method described above. A recently developed perfectly matched layer (PML) technique that mitigates the effect of spurious reflections from the inflow and outflow computational boundaries is also applied [32]). Details of the space-time DG method can be found elsewhere [32-34] but are briefly summarized here for the sake of completeness. The spatial domain, $\Omega$, is partitioned into non-overlapping hexahedral elements, $\kappa$, while time is partitioned into time intervals (or time-slabs), $I^{n}=\left[t^{n}, t^{n+1}\right]$. After defining $\mathscr{V}_{h}=\left\{\boldsymbol{w},\left.\boldsymbol{w}\right|_{\kappa} \in[\mathscr{P}(\kappa \times I)]^{5}\right\}$, the space-time finiteelement space is discretized using piece-wise polynomial functions in both space and time. The numerical flux functions approximating the inviscid, viscous, and PML fluxes at the spatial boundaries of the elements are computed using the entropystable flux function of Ismail and Roe [35], the Bassi and Rebay operator [36], and a fully upwind method, respectively. We seek a solution $v \in \mathscr{V}_{h}$ that satisfies the weak-form equation for all $\boldsymbol{w} \in \mathscr{V}_{h}$. The space $\mathscr{V}_{h}$ is spanned by the tensor product of 1D nodal Lagrange basis functions defined at the Gauss-Legendre points. Integrals in the weak-form equation are approximated with a quadrature rule using twice the number of quadrature points as solution points in each spatial coordinate and temporal directions in order to minimize quadrature errors. The resulting nonlinear system of equations is then solved using a preconditioned Jacobian-free Newton-Krylov solver [33]. It is noted that using entropy variables and these choices of flux functions ensures discrete entropy stability for the Navier-Stokes equations in the DG formulation [30].

\section{INFLOW TURBULENCE GENERATION RESULTS}

The linear forcing method for generating inflow turbulence was first evaluated by simulating HIT at $R e_{\lambda}=45$ and a turbulent Mach number of 0.1 in a unit cube with 32 elements in each direction, and using the spatially 8th-order (i.e., 256 degrees of 

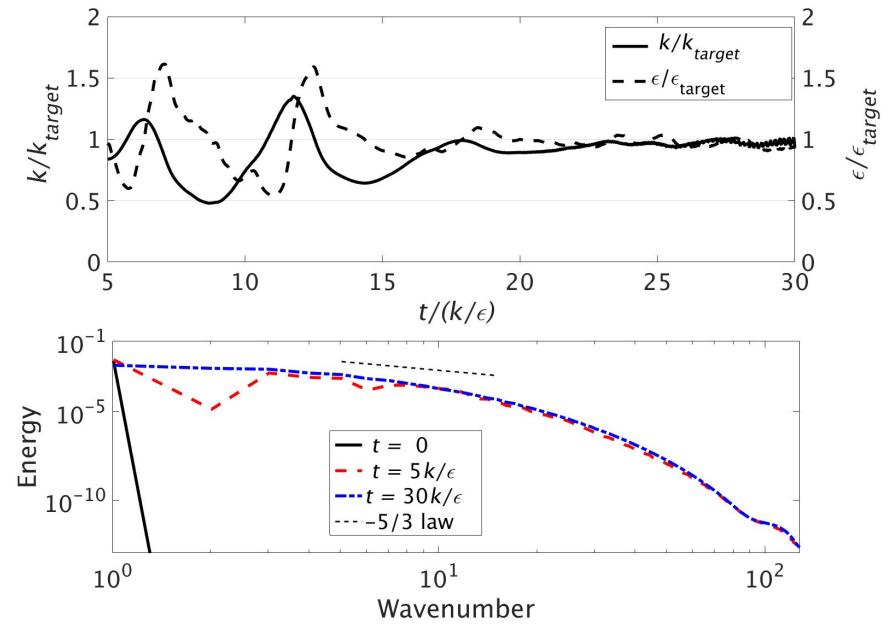

FIGURE 1: TURBULENT KINETIC ENERGY AND DISSIPATION EVOLUTION (TOP PANEL) AND TURBULENT ENERGY SPECTRUM (BOTTOM PANEL) FOR $R e_{\lambda}=45$.

freedom in each spatial direction) and temporally 4th-order DG scheme. Instead of using an idealized turbulent spectrum as the initial condition, the flow field is initiated using a Taylor-Green vortex [37]. This vortex initially undergoes vortex stretching and then breaks down to turbulence. The linear forcing method prevents the eventual decay of the Taylor-Green vortex and instead evolves the flow field to the desired turbulent state. The inputs to the linear forcing technique are chosen as described earlier: the desired $\varepsilon$, estimated using a turbulent Mach number of 0.1 , and an integral length scale that is assumed to be $20 \%$ of the size of the computational domain.

The flow attains the turbulent stationary state with the desired target turbulent kinetic energy and dissipation after $20 k / \varepsilon$ (Fig. 1). Small high-frequency oscillations in the turbulent kinetic energy are noticed (discernable by zooming into Fig. 1 in the region beyond $25 k / \varepsilon$ ) as the simulation continues for longer eddy turnover times. These arise from the highly oscillatory $P D$ term (although the time-averaged stationary $P D$ is almost zero). Similar high frequency $P D$ oscillations have also been noticed in previous compressible forced homogeneous simulations [38-40]. The velocity spectrum evolves to the full spectrum after the flow attains stationarity (Fig. 1). The Reynolds normal stresses converge to the targeted value, and the Reynolds shear stresses converge to zero. The longitudinal velocity gradients are half the transverse velocity gradients in the stationary state and these values are consistent with incompressible HIT.

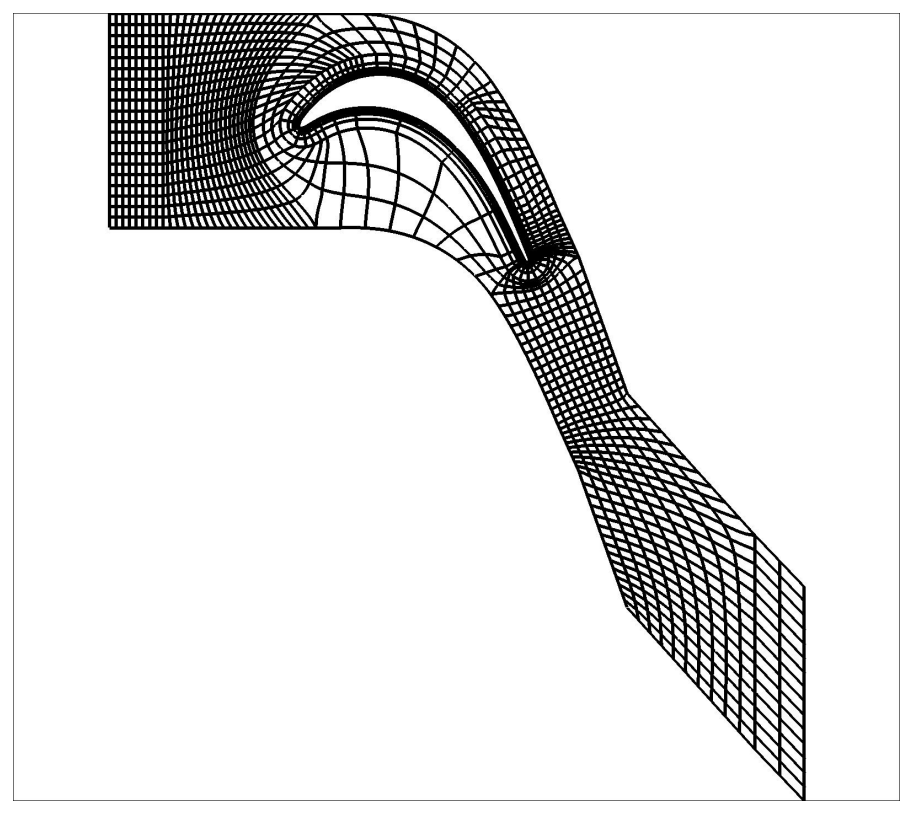

FIGURE 2: A REPRESENTATIVE COMPUTATIONAL MESH WITH 8TH-ORDER ELEMENTS.

\section{T106A LOW PRESSURE TURBINE CASCADE}

The lightly loaded T106A LPT cascade has been studied extensively both in experiments $[41,42]$, and numerical simulations $[3,9,10,18$, and the references cited therein]. The T106 profile represents the mid-span section of the Pratt and Whitney PW2037 rotor airfoil. The airfoil pitch for this configuration is $0.799 C$, where $C$ is the airfoil axial chord length. The exit isentropic Reynolds number is chosen as 60,000 and the exit isentropic Mach number as 0.405 from the experiment [41] for the case without any simulated upstream wakes. The inlet flow angle is chosen as $45.5^{\circ}$ to be consistent with previous numerical studies but differs from the value noted in the experiment $[3,8,9]$. Two different inflow turbulence levels, $0 \%$ (clean inflow) and $2 \%$, are considered.

Mid-span section of single passage of the cascade is meshed using same procedure as described in [18]. Fig. 2 shows a representative mesh constituting 8th-order elements. At the upstream region of the blade, refined mesh is used to simulate inflow turbulence effect. The spanwise extent of the computational domain is chosen as $0.20 \mathrm{C}$ in the present study. Note that periodic boundary conditions are used in both the pitch and span directions.

For the clean inflow simulations, buffer regions with streamwise length of $0.20 C$ are used for the PML technique both at the inflow and outflow regions. The PML technique helps eliminate any spurious reflection from the boundaries [32]. Note that a small velocity fluctuation level, $0.6 \%$, was measured at the inflow region in the experiment even with clean inflow. A compu- 
tational mesh with 130328 th-order spatial elements $\left(6.6 \times 10^{6}\right.$ degrees of freedom) and 4th-order temporal resolution is chosen for present study, based on $h-p$ convergence studies performed previously [18].

To simulate 2\% inflow turbulence, the inflow PML buffer region is converted to the inflow turbulence generating blocks while the outflow buffer region is maintained as before. Four periodic cubes with dimension equal to the spanwise domain are used for the present simulations (Figs. 6b). The fully developed turbulence is then (one-way) coupled to the main computational domain by solving a numerical Riemann problem at the inflow boundary face of the main computational domain (located $0.5 C$ upstream of the leading edge). Note that the HIT generating cubes are decoupled from the main DNS domain to ensure that upwind-traveling acoustic perturbations from the main domain do not introduce unwanted energy into the turbulence generation process. In a manner similar to the HIT simulations described above, the velocity field in the turbulence generating blocks are initiated using a Taylor-Green vortex superimposed with the inflow condition. Since we are not enforcing the total energy Eqn. [2c], the strength of the Taylor-Green vortex is adjusted to achieve the target inflow static pressure when the flow attains a statistically stationary state.

As mentioned earlier, the resulting integral length scale is $20 \%$ of the cube dimension for linear forced turbulence. For our case this is $0.04 C$, resulting in a $R e_{\lambda}$ of 20 with $2 \%$ inflow turbulence at the turbulence generating blocks. The effective degrees of freedom for each block is $64 \times 40 \times 64$. In order to achieve higher integral length scales, the spanwise extent of the computational domain would need to be increased. Although similar initial conditions and forcing are used in the four turbulence generating blocks, the velocity field in each block is different at the stationary state (Fig. 6b-ii). By using four separate turbulent generating blocks instead of one we have avoided introducing any unwanted large scale periodicity in the pitch direction. To minimize additional numerical dissipation of the inflow turbulence as it advects downstream, higher resolution (similar to the turbulence generating blocks) is used (Fig. 2) in the region upstream of the airfoil. As the numerically generated turbulence approaches the airfoil it decays to $1.8 \%$ at $x=0$ plane. The experimental data for the T106A configuration does not include any details regarding the inflow turbulence.

\section{Numerical Results}

We first compare the mean flow statistics - surface pressure, friction coefficient, and wake loss - with the experimental data [41], and prior numerical simulations $[3,8,9]$. These flow statistics are computed by averaging both in span and in time after stationarity is achieved. Flow stationarity was achieved after three domain flow-through times and is assessed by monitoring the temporal evolution of the integrated surface force on the air-

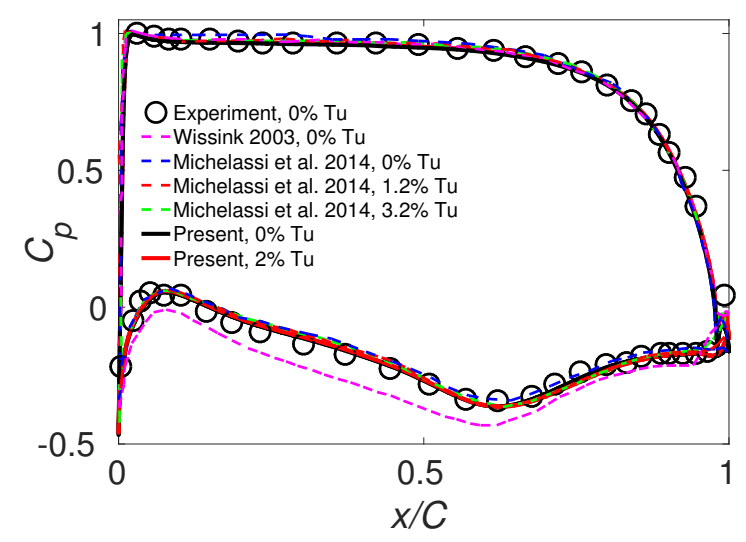

FIGURE 3: COMPARISON OF AIRFOIL LOADINGS FOR THE T106A CASCADE.

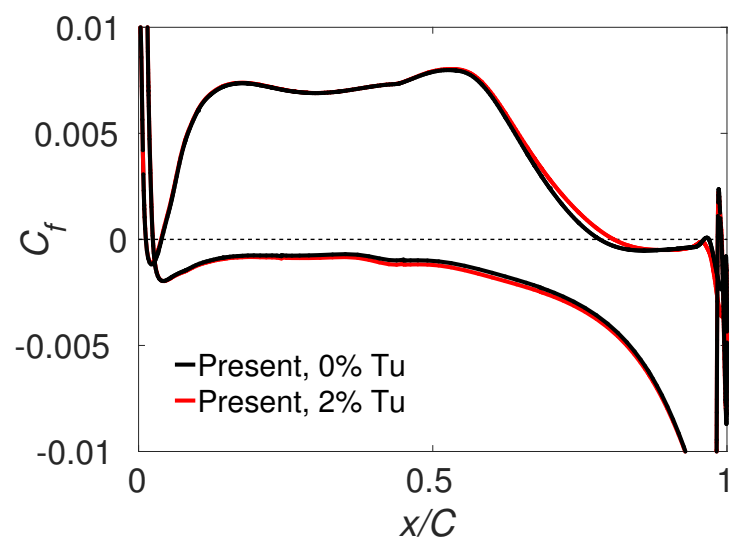

FIGURE 4: COMPARISON OF WALL SHEAR-STRESS COEFFICIENT FOR THE T106A CASCADE. DASHED BLACK LINE REPRESENTS $C_{f}=0$.

foil. The flow statistics are computed over five domain flowthrough times.

With clean inflow, the surface pressure distribution compares well with the experiment [41] and a recent compressible DNS simulation $[8,9]$ using a 4th-order finite-difference approach (Fig. 3). Incompressible DNS [3] predicts a much lower suction pressure, and this mismatch is attributed to the effects of compressibility. A $2 \%$ inflow turbulence level does not influence the airfoil loading except very close to the trailing edge (Fig. 3) where the suction-side surface pressure increases marginally. Michelassi et al. [10] also noted similar behavior in the $C_{p}$ distribution when comparing $1.2 \%$ and $3.2 \%$ inflow turbulence levels, although they reported higher pressure values than the present 


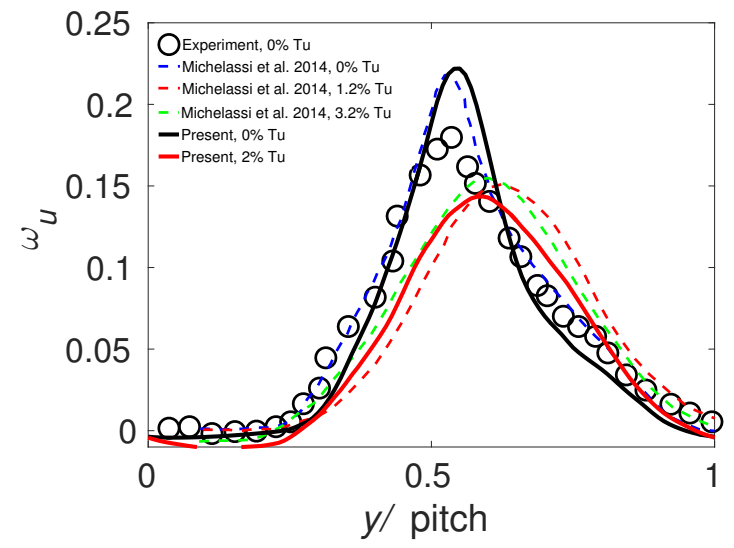

FIGURE 5: COMPARISON OF WAKE LOSS PROFILES FOR THE T106A CASCADE.

simulations for both the $1.2 \%$ and $3.2 \%$ inflow turbulence cases studied.

The shear stress profiles indicate the presence of two separation bubbles on the suction surface (Fig. 4). The size of the leading-edge separation bubble remains insensitive to the inflow turbulence level but the size of the aft separation bubble shows minor changes when inflow turbulence is present. The location of the aft flow separation point shifts from $0.78 \mathrm{C}$ for clean inflow to $0.8 C$ with $2 \%$ inflow turbulence. The flow on the pressure side remains attached independent of inflow turbulence level in agreement with prior observations [10].

The wake deficit $\left(\omega_{u}=\frac{p_{t 1}-p_{t}}{p_{t 1}-p_{2}}\right)$ is computed at a location $0.4 C$ downstream of the airfoil trailing edge. Despite the good agreement between the simulations and the experimental surface pressures, the clean inflow simulation over-predicts the wake loss as compared to the experiment but is similar to other DNS [10] (Fig. 5). Note that since the experiments do not completely document the pitch location of the peak, the numerical wake loss profiles in the figure have been shifted to match the experimental peak location. The reason for the mismatch in the wake deficit profiles is unclear, but may be related to the small turbulence intensity of $0.6 \%$ noted in the experiments even with clean inflow. When the inflow turbulence level is increased, the laminar separation bubble shrinks (Figs. 6b, 4), wake losses are reduced, and the outflow angle changes (Fig. 5). The wake loss has been noted [10] to be unchanged when the inflow turbulence was increased from $1.2 \%$ to $3.2 \%$, and and our estimated wake loss with inflow turbulence case compares well with these results.

The transient flow-field is investigated using instantaneous Mach number (Fig. 6i) and vorticity magnitude (Fig. 6ii) at the mid-span location. On the suction side, the flow accelerates up to $x<0.6 C$, then decelerates and separates in the aft region. The flow remains laminar and attached on the pressure side (Fig. 6i).
The aft separation bubble moves the shear layer away from the suction surface (Fig. 6ii). Transition to turbulence occurs in the separated shear layer very close to the trailing edge on the suction side. Laminar vortex shedding from the pressure-surface shear layer undergoes laminar roll-up, whereas on the suction side the vortex roll-up is turbulent. The laminar and turbulent shear layers merge downstream of the airfoil trailing edge to form a fully turbulent wake.

The presence of inflow turbulence (Fig. $6 \mathrm{~b}$ ) reduces the size of the suction-side separation bubble and influences the vortex shedding process. The pressure-side boundary layer still remains laminar. Turbulent vortices undergo strong stretching at the airfoil leading edge (Fig. 6b-ii). These distorted inflow vortices perturb the suction-side separated shear layer and merge with the airfoil wake downstream of the trailing edge.

Details of the flow transition process are studied using the isosurfaces of the vorticity magnitude over the suction side of the airfoil. The inflow turbulence perturbs the leading edge separation bubble (Fig. 7b-ii) to generate long streamwise coherent structures. These structures survive the flow acceleration at the suction peak, and perturb the separated shear layer to generate a spanwise sinusoidal distortion (Fig. 7b-i) close to the trailing edge. With clean inflow, no similar structures originate from the leading edge separation bubble (Fig. 7a-i). The separated shear layer transitions to turbulence through a Kelvin-Helmholtz type roll-up (Figs. 6ii, 7) close to trailing edge for both cases with and without inflow turbulence. The spanwise sinusoidal waves also influence the flow transition process for non-zero inflow turbulence levels.

The turbulence generation process through the growth of the unstable modes, as seen in Fig. 7, is further investigated using the maximum value of turbulent intensity ( $\mathrm{Tu}\left(=\frac{\sqrt{\frac{1}{3}\left(u^{\prime} u^{\prime}+v^{\prime} v^{\prime}+w^{\prime} w^{\prime}\right)}}{M a_{i s 2}}\right)$ in the wall-normal direction, and the spanwise anisotropy $\left(b_{33}=\right.$ $\left.\frac{w^{\prime} w^{\prime}}{2 k}-\frac{1}{3}\right)$ at the maximum Tu location as a function of the streamwise distance (Fig. 8) in the aft region of the suction side. For both cases with and without inflow turbulence, the maximum value of $\mathrm{Tu}$ is negligible prior to flow transition. The $2 \%$ inflow case has slightly higher values of $b_{33}$ compared to the clean inflow case before flow transition due to the presence of streamwise flow structures (Fig. 7b-ii). As the flow transitions close to the trailing edge, the maximum $\mathrm{Tu}$ is seen to increase rapidly, and the coherent disturbances break down into turbulence resulting in higher $b_{33}$ values. The transition process starts at the same location for both cases. Based on the Tu growth near the trailing edge of the airfoil, it appears that the transition process has not yet completed before the trailing edge. Also note that the maximum value of $\mathrm{Tu}$ is attained inside the boundary layer for both inflow turbulence cases.

The wall normal profiles of streamwise velocity $\left(\frac{u_{\xi}}{M a_{i s 2}}\right), \mathrm{Tu}$, and turbulence production and dissipation are plotted at seven monitoring stations (Fig. 9). Station 'A' is located close to the 
leading edge, while station ' $\mathrm{B}$ ' is located just past the accelerated flow region. The remaining stations are located in the separated bubble region. Note that the stations ' $F$ ' and ' $G$ ' are beyond the flow transition region (Figs. 7, 8). As the flow accelerates in the fore region of the airfoil, the boundary-layer remains laminar and its thickness changes only marginally. Beyond the suction-side peak location, the flow decelerates to form a separation bubble and the boundary-layer thickness increases rapidly (Fig. 9i). For the clean inflow simulations, the turbulence intensity and hence the turbulence production is negligible for $x<0.95 C$ (Figs. 8, 9ii, 9iii). For non-zero inflow turbulence, the inflow turbulent vortices undergo strong stretching (Fig. 6bii), resulting in larger turbulence intensities (Fig. 9ii) close to the leading edge. These are manifested as streamwise structures (Fig. 7b) in the flow field. These streamwise disturbances (Fig. 7b) result in larger turbulence intensities compared to the clean inflow case (Fig. 9ii). Inflow turbulence reduces the suction-side boundary layer thickness (Fig. 9i) in the aft region. For both clean and non-zero inflow turbulence, most of the turbulence is generated in the separated shear layer (Fig. 9iii) close to the trailing edge.

As the flow accelerates through the suction peak (Fig. 6), high turbulence dissipation is noted at stations ' $\mathrm{A}$ ' and 'B'. However, the turbulence dissipation remains small compared to the turbulence production term during flow transition in the separated shear layer irrespective of the inflow turbulence level (Fig. 9iii).

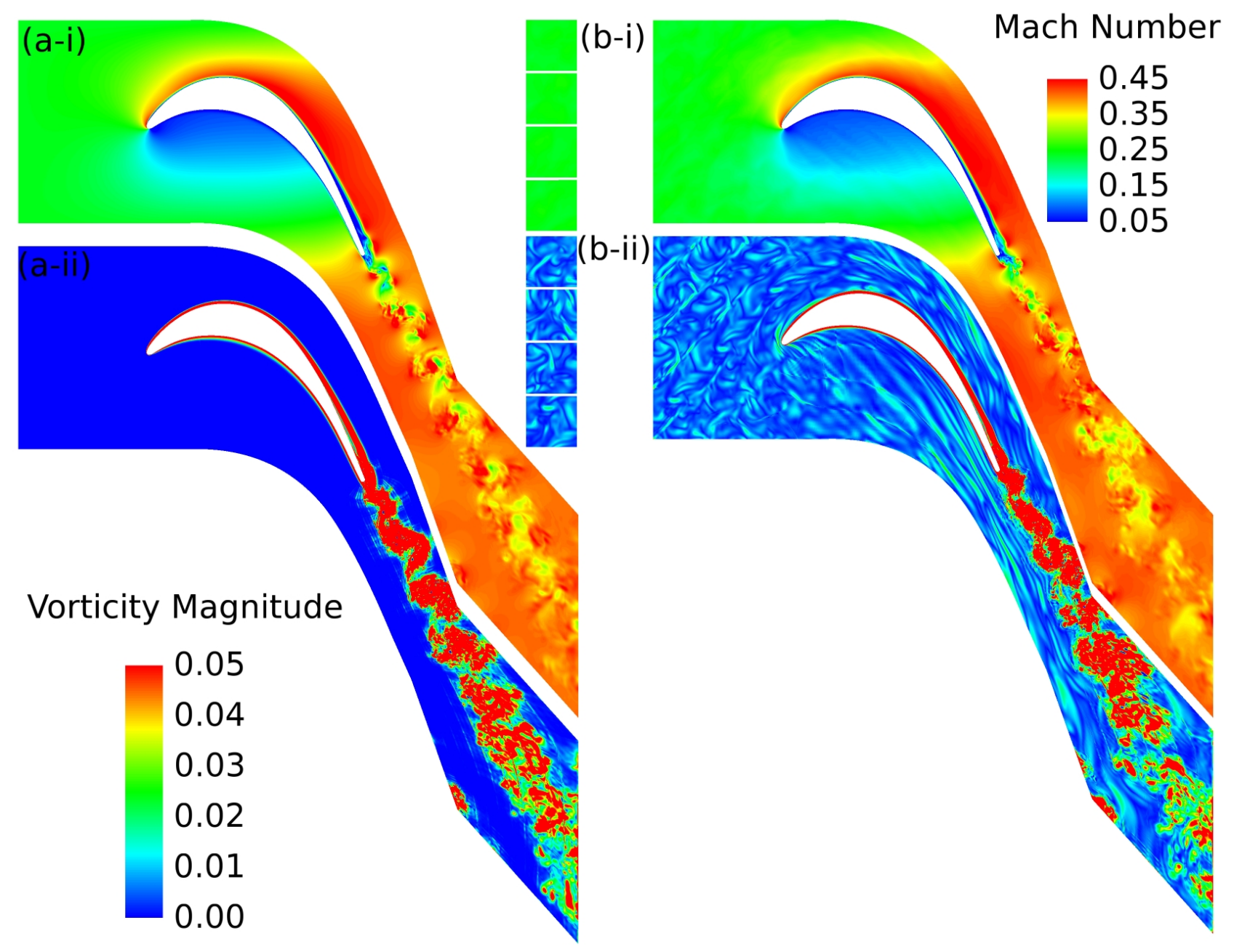

FIGURE 6: INSTANTANEOUS MID-SPAN (i) MACH NUMBER, AND (ii) VORTICITY MAGNITUDE CONTOURS FOR THE T106A CASCADE FOR (a) CLEAN INFLOW, AND (b) 2\% INFLOW TURBULENCE. 

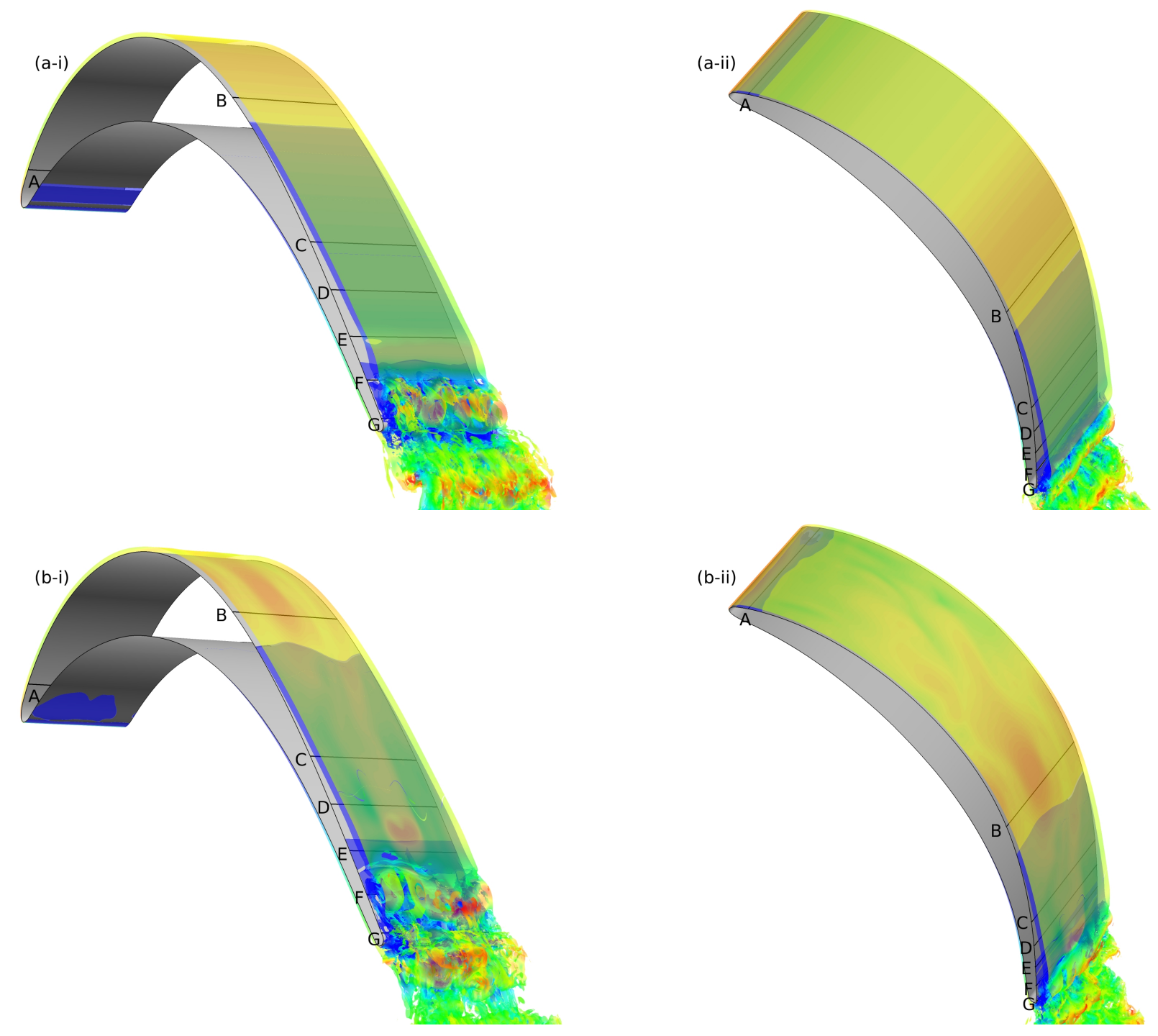
FIGURE 7: ISOSURFACES OF VORTICITY MAGNITUDE FOR THE T106A CASCADE FOR (a) CLEAN INFLOW, AND (b) $2 \%$
INFLOW TURBULENCE. DIFFERENT PERSPECTIVE VIEWS ARE INCLUDED IN (i) AND (ii) FOR CLARITY.

\section{T106C LOW PRESSURE TURBINE CASCADE}

In the T106C configuration the airfoil pitch is increased to $0.95 \mathrm{C}$ and results in higher airfoil loading and the formation of a large separation bubble in the aft region of the suction surface. This configuration has been studied extensively for different inflow turbulence levels and exit isentropic Reynolds numbers [1]. These studies have found that the suction-side separation bubble topology changes from long to short as the inflow turbulence level and Reynolds number are increased (Table 3 in [1]). In this study, the exit isentropic Reynolds number is chosen as 80,000 and two cases are considered, clean inflow and and 3.2\% inflow turbulence, for which changes in the separation bubble topology have been reported [1]. The exit isentropic Mach number and inflow angle are chosen as 0.65 and $32.7^{\circ}$, respectively. The Reynolds number $=80,000$ case has also been studied numerically by others $[16,17]$ for clean inflow conditions.

Note that in the experiments the "clean" inflow turbulence case has a non-zero background turbulence level of $0.9 \%$ and an integral length scale corresponding to $0.32 \mathrm{C}$. Duplicating the experimental $R e_{\lambda}=240$ in the computations would require a spanwise dimension of $1.6 \mathrm{C}$ since the linear forcing technique requires a domain size that is about 5 times the integral length scale. This spanwise dimension is about the same order as the spanwise extent of the experimental airfoils, 2.4C. In other 

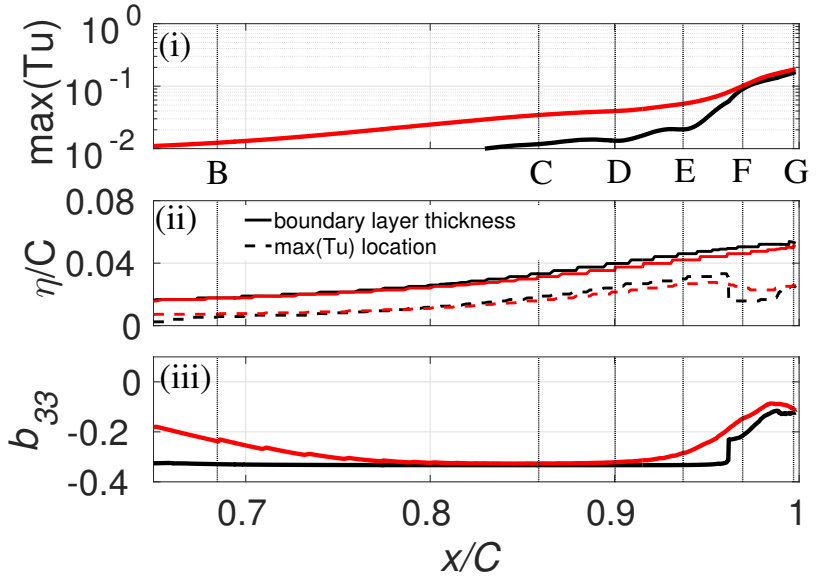

FIGURE 8: COMPARISON OF (i) Tu GROWTH RATE, (ii) BOUNDARY LAYER THICKNESS, MAXIMUM Tu LOCATION, AND (iii) $b_{33}$ FOR THE T106A CASCADE FOR CLEAN INFLOW (BLACK LINE) AND 2\% INFLOW TURBULENCE (RED LINE).

words, the entire airfoil passage would have to be simulated. Similarly, for the $3.2 \%$ inflow turbulence case, the integral length scale in the experiment was reported as $0.13 C$ (experimental $R e_{\lambda}=98$ ), requiring a spanwise domain extent of 0.65C. However, in order to keep the computational cost reasonable, we have chosen the spanwise domain extent to be much smaller, $0.24 C$ for both the $0 \%$ and $3.2 \%$ inflow turbulence cases. The elements for the DG solver are generated following the same procedure as described above for the T106A configuration.

As in the case of the T106A cascade, for the clean inflow simulation we have added two buffer regions of streamwise length of $0.24 C$ in the inflow and outflow regions of the domain to implement the PML boundary technique. We have modified the T106A elements (Fig. 2) to increase the pitch dimension, and have reduced the number of elements from 3 to 2 in the wall-normal direction at the off-body O-topology blocks for the T106C configuration. $p$ convergence studies conducted on the resulting mesh showed marginal improvement with 8thorder compared to using 4th-order discretizations. Hence, for the T106C simulations reported here we have used a mesh with 12464 8th-order spatial elements and 4th-order temporal resolution.

The $3.2 \%$ inflow turbulence case for this configuration uses slight modifications to the T106A $2 \%$ inflow turbulence procedure. In the T106A inflow turbulence setup, we used four turbulence generating cubes that are physically separated from one another. When the generated turbulence is fed into the main computational domain, discrete jumps are noticed across the cube boundaries (Fig. 6bii). In order to minimize these numerical
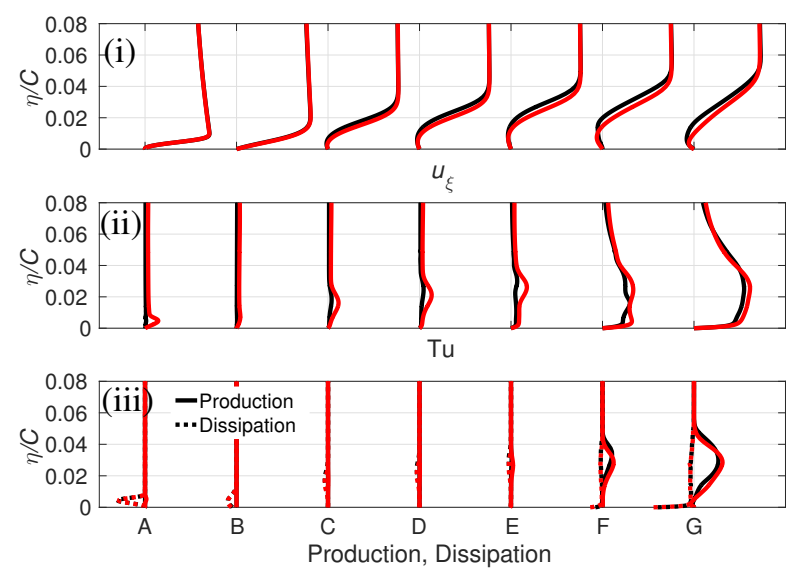

FIGURE 9: WALL NORMAL PROFILE OF (i) MEAN TANGENTIAL VELOCITY, (ii) TURBULENT INTENSITY, AND (iii) TURBULENT PRODUCTION AND DISSIPATION AT THE MONITORING STATIONS, AS SHOWN IN FIG. 7, ON THE AIRFOIL SUCTION-SIDE FOR THE T106A CASCADE FOR CLEAN INFLOW (BLACK COLORED LINE), AND 2\% INFLOW TURBULENCE (RED COLORED LINE).

artifacts, the turbulence generating cubes for theT106C inflow turbulence case are stacked physically adjacent to one another (Fig. 13bii).

The turbulence generating cubes are located $0.5 C$ upstream of the leading edge of the airfoil. Following the decay law [1], we estimate the turbulence intensity to be $3.5 \%$ at the turbulence blocks in order to achieve a $3.2 \%$ turbulence intensity level at the $x=0$ plane (although our integral length scale differs from the experimental value as noted above). This results in a $R e_{\lambda}=30$. Since we are using $64 \times 40 \times 64$ degrees of freedom for each of the cubes, the generated HIT is under-resolved, and has a higher turbulence intensity level (3.8\%) compared to the target. This turbulence advects toward the airfoil and decays to the $3.5 \%$ level at the $x=0$ plane.

\section{Numerical Results}

The isentropic Mach number, skin friction coefficient, and wake deficit are first compared with the experiment [1] and previous DNS [16]. These flow statistics are gathered during the stationary period. By monitoring the temporal evolution of the integrated airfoil surface force the flow is seen to attain stationarity after three domain flow-through times. Flow statistics are obtained by averaging both in span and in time over three domain flow-through times beyond the stationary state.

Although the predicted airfoil loadings on the pressure side agree well with the experiment, the surface loadings in the fore region of the suction side are over-predicted, similar to the re- 
sults in prior numerical studies [16]. Hillewaert et al. [16] conjectured that this mismatch may be due to incorrect settings of the inflow angle and airfoil stagger angle. Beyond the suction peak in the laminar separation region, the present results are in better agreement with the experiments than the earlier study [16]. This is due to the use of the inflow and outflow PML technique; our results without the PML inflow and outflow boundary conditions closely matched these earlier computational results. The PML buffer regions eliminate almost all spurious boundary reflections, resulting in a better prediction of the laminar separation bubble [32]. However, the transition location is still not accurately captured (Fig. 11), and results in a different surface pressure distribution than the experiment in the region close to the trailing edge. We are currently investigating the surface pressure mismatch issue by modifying the inflow angle, as in [16]. Note that the inflow turbulence does not affect the airfoil loading except in the aft region of the suction side. The inflow turbulence results in a higher isentropic Mach number further downstream on the suction side and higher pressures close to the trailing edge as compared to the clean inflow case.

Unlike for the T106A cascade(Fig. 4), there is no leading edge suction-side separation bubble for the T106C configuration (Fig. 11) with or without inflow turbulence. Flow separation occurs at $0.67 C$ on the suction side for the clean inflow case and moves downstream to $0.74 C$ with inflow turbulence; and flow nominally reattaches at $0.96 \mathrm{C}$ after undergoing transition. As in the T106A case, the pressure-side boundary layer remains attached for both cases with and without inflow turbulence.

For the clean inflow case, the mismatch in the surface pressure is also reflected in the wake loss profiles $\left(\omega_{u}=\frac{p_{t 1}-p_{t}}{p_{t 1}}\right)$ prediction (Fig. 12) when compared to the experiment at a location $0.465 C$ downstream of the trailing edge. As was done for the T106A case, the numerically estimated wake loss profile have again been shifted to match the peak location. The peak wake loss is under-predicted by $30 \%$. Prior simulations by [16] also under-predicted the peak loss, but our result is closer to the experimental data. With non-zero inflow turbulence, the peak wake loss and the wake thickness are smaller compared to the clean inflow case due to the flow reattaching downstream. Similar results were noted for the T106A simulations with and without inflow turbulence (FIg. 5).

Similar to the T106A clean inflow simulation, the flow accelerates up to $0.6 \mathrm{C}$ and remains laminar over the fore region of suction surface. As the flow decelerates downstream of the suction peak, a laminar separation bubble forms (Fig. 13a), which is considerably bigger than for the T106A clean inflow case (Fig. 6a). The separated shear layer moves away from the airfoil surface, and transition to turbulence occurs near the airfoil trailing edge (Figs. 13a). Inflow turbulence decreases the suctionside separation bubble considerably (Fig. 13b). The suction-side boundary layer moves closer to the airfoil surface and results in a thinner wake downstream of the trailing edge(Figs. 13b). The

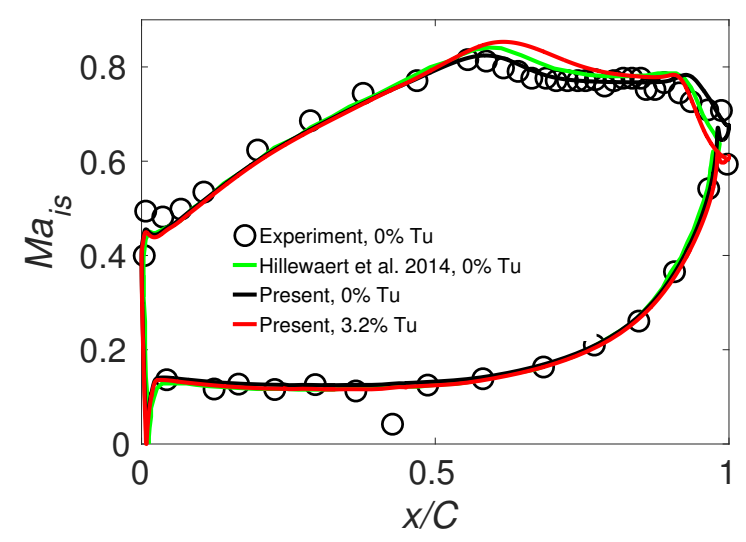

FIGURE 10: COMPARISON OF AIRFOIL LOADINGS FOR THE T106C CASCADE.



FIGURE 11: COMPARISON OF WALL SHEAR STRESS COEFFICIENT FOR THE T106C CASCADE. DASHED BLACK LINE REPRESENTS $C_{f}=0$.

flow on the pressure side is laminar and continues to remain attached, again as in the T106A case (Fig. 11). Downstream of the airfoil, fully turbulent wake shedding is noticed (Fig. 13ii)

The turbulent flow structures are studied using 3D vorticity magnitude isosurface contours. As in the T106A case (Fig. 7), the separated shear layer undergoes Kelvin-Helmholtz-type rollup (Figs. 13ii, 14) both with and without inflow turbulence. During flow transition, streamwise oblique flow structures also appear above the Kelvin-Helmholtz mode (Fig. 14a-ii). The non-zero inflow turbulence perturbs the separated shear layer by generating streamwise coherent flow structures (Figs. 14b-i) that further energize the oblique flow structures during the transition process (Fig. 14b-ii). 


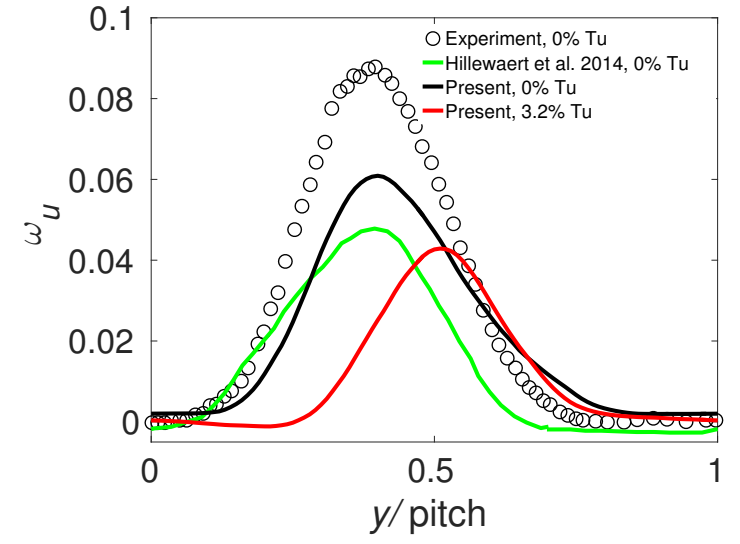

FIGURE 12: COMPARISON OF WAKE LOSS PROFILES FOR THE T106C CASCADE.

The transitional behavior of the separated boundary layer can be studied by looking at the growth rate of Tu in the aft region of the suction surface (Fig. 15). For both clean and 3.2\% inflow turbulence cases, the Tu value initially grows, attains a maximum, and then decays with streamwise distance. The growth rate of Tu is higher over the laminar part $(0.7 C \lesssim x \lesssim 0.9 C)$ of the separation bubble for the clean inflow case, although the spanwise component is almost zero. Figs. 13a-ii, 14a-i indicate that the turbulence mode is of the Kelvin-Helmholtz type. For the 3.2\% inflow turbulence case, long streamwise structures are noticed (higher spanwise component compared to the clean inflow, but still small compared to the other components). During the transition process, these structures break down into three-dimensional turbulence with similar growth rate of Tu for both the inflow cases. The transition process is initiated at the same location for both inflow turbulence cases, but it terminates earlier for the $3.2 \%$ inflow turbulence due to flow reattachment (Fig. 11). As in the T106A case, the location of maximum Tu lies inside the boundary layer for both inflow turbulence cases. Streamwise structures are also noticed on the pressure side for the non-zero inflow case, although they do not appear to transition to 3D turbulent structures.

The mean tangential velocity, turbulence intensity, turbulence production and dissipation profiles over the suction surface are plotted at the same monitoring stations as for the T106A case. Note that monitoring stations beyond ' $\mathrm{D}$ ' are downstream of flow transition (Figs. 14, 15) for the current T106C case. A large suction-side separation bubble is evident from the mean tangential velocity profiles with reverse flow for the clean inflow case. As the flow separates the boundary layer thickness increases rapidly in the aft region of the airfoil (Fig. 16i). The suction-side boundary layer remains separated even beyond flow transition (Fig. 16i). As in the T106A case, the Tu value peaks
(Fig. 15) inside the shear layer (Fig. 16ii), and most of the turbulence is produced in the separated shear layer (Fig. 16iii) through Kelvin-Helmholtz roll-up (Fig. 14a).

Changes in the separation bubble topology, from long to short, due to inflow turbulence can be observed in the mean tangential velocity profiles (Fig. 16i). Flow separation is delayed with inflow turbulence, and the flow is seen to reattach after transition. This causes the size of the separation bubble to decrease by almost a factor of 2 , and the boundary layer thickness to be much smaller (Fig.16i). Inflow turbulence perturbs the shear layer to generate streamwise structures (Fig. 14b-ii), resulting in almost constant turbulence intensity over wall normal distance between the ' $\mathrm{A}$ ' and ' $\mathrm{B}$ ' monitoring stations. Inflow turbulence also alters the turbulence intensity profile in the aft region (Figs. 16ii). Although the turbulence intensity peaks at the separated shear layer over the separation bubble similar to the clean inflow case, it remains almost constant after flow reattachment over the lower part of the boundary layer. This behavioral change of turbulence intensity is also reflected in the turbulence production term (Fig. 16iii). The turbulence production peaks at the separated shear layer over the separation bubble but turbulence production inside the shear layer begins to drop as the flow reattaches, and another peak very close to the wall develops.

As in the T106A case, the turbulence dissipation overwhelms the production in the fore region of the airfoil, and is much smaller compared to the turbulence production in the aft region for both cases with and without inflow turbulence (Fig. 16iii). Although the mean tangential velocity profile looks smooth at the element boundaries, large jumps in higher-order moments such as turbulence intensity, turbulence production, and turbulence dissipation are still noticeable at the element boundaries, signifying inadequate mesh resolution to capture these high-order moments.

\section{SUMMARY AND CONCLUDING REMARKS}

Results from DNS of transitional and turbulent flow in the T106A and T106C linear turbine cascades at elevated inflow turbulence levels using a high-order entropy-stable DG spectralelement method with a space-time formulation are presented. The inflow turbulence is generated in the simulation using a linear forcing technique adapted for the DG framework. The PML technique is also used to mitigate spurious numerical reflections from the boundaries of the computational domain. Thanks to the 8 th-order spatial discretization $\left(6.6 \times 10^{6}\right.$ degrees of freedom), the quality of our solutions is similar in comparison to the previous 4th-order spatial DNS for T106A [9] $\left(18.1 \times 10^{6}\right.$ degrees of freedom), and better in comparison to the previous 4 th-order DNS for T106C [16] $\left(16.8 \times 10^{6}\right.$ degrees of freedom) with much less degrees of freedom.

The effects of inflow turbulence levels on the suction-side separation bubble in both the T106A and T106C cascades are 


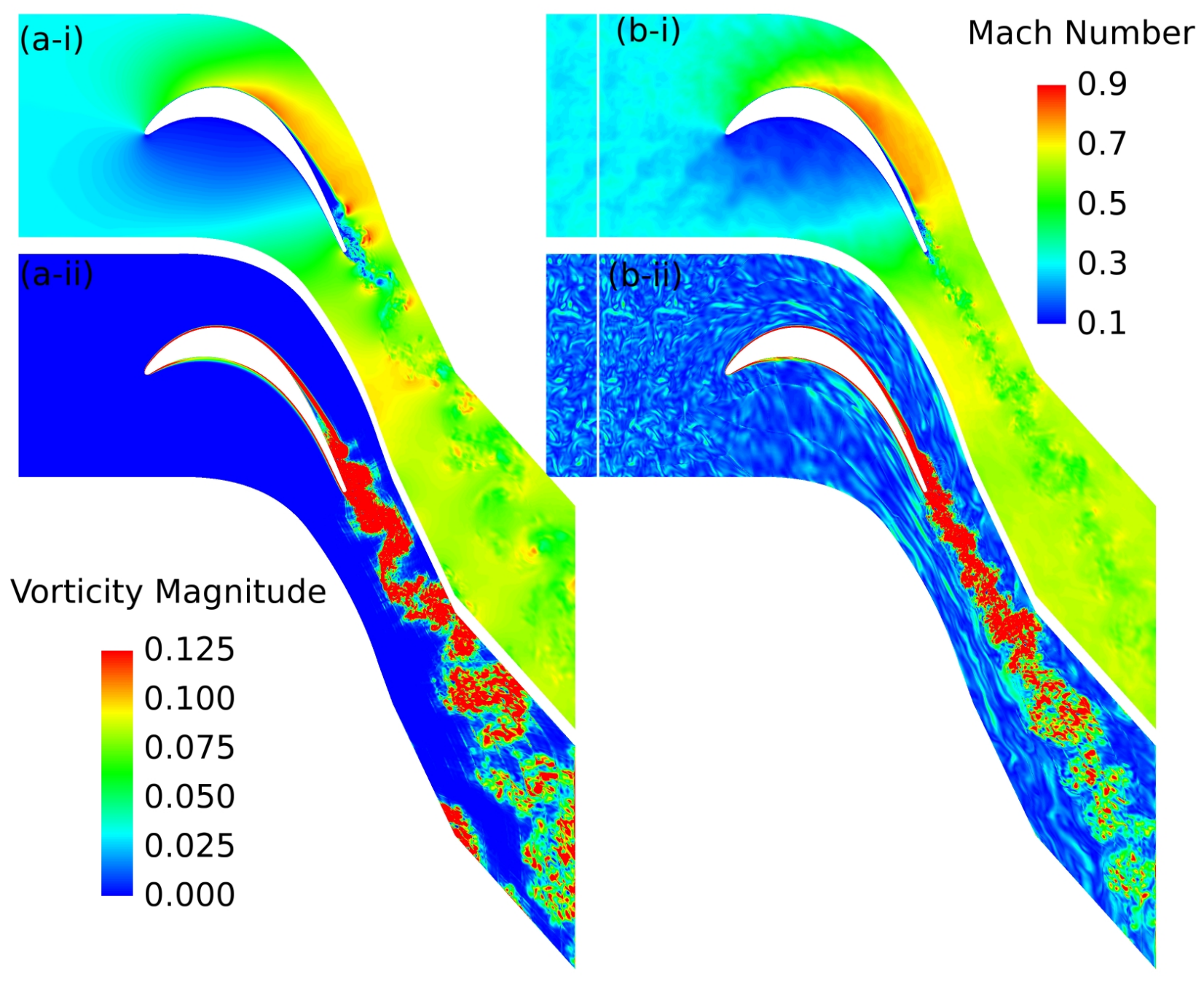

FIGURE 13: INSTANTANEOUS MID-SPAN (i) MACH NUMBER, AND (ii) VORTICITY MAGNITUDE CONTOURS FOR THE T106C CASCADE FOR (a) CLEAN INFLOW, AND (b) $3.2 \%$ INFLOW TURBULENCE.

studied. These results show that even maintaining the same airfoil section with small differences in loading, the inclusion of inflow turbulence is important and the detailed effects are individual for each configuration. For the clean inflow T106A simulation, the airfoil surface pressure loadings agree well with experiment and with previous compressible DNS studies. For the T106C cascade, the airfoil surface pressure loadings at the fore section are over-predicted, though this result is expected as similar observation was made in prior DNS simulations by other authors. With the inclusion of the PML buffer outflow region the surface pressures approach the experiment values over the separation bubble, but the transition characteristics close to the trailing edge are not in agreement. Hillewaert et al. [16] conjectured that the loading mismatch in the fore section of the suction-side of the airfoil may be related to discrepancies in the inflow angle between the experiment and the simulations. The predicted wake loss profiles for the T106A cascade are similar to earlier reported work [10], and for the T106C cascade are similar to [16] for clean inflow conditions but our results are somewhat closer to the experiment, thanks to the PML. The inflow turbulence drops the peak in the wake loss profile and the wake thickness for both configurations are lower in the presence of inflow turbulence. In addition to the suction-side separation bubbles noted on the aft regions of both cascades, a leading edge separation bubble is observed near the leading edge of the T106A cascade.

For the T106A configuration with $R e=60,000$, a $2 \%$ inflow turbulence level has a minor effect on the topology of the suction-side bubble. The airfoil surface pressures remain insen- 

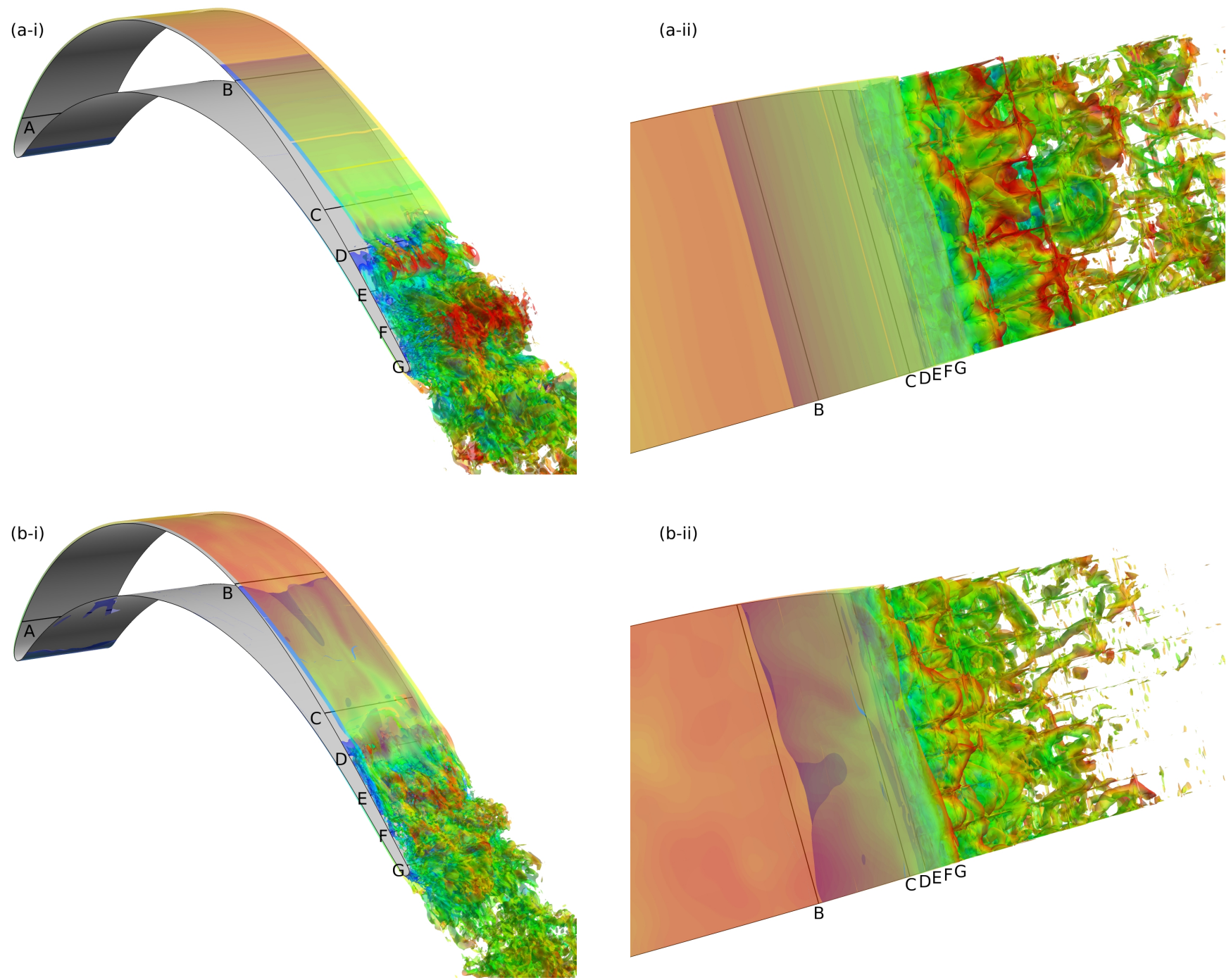

FIGURE 14: ISOSURFACES OF VORTICITY MAGNITUDE FOR THE T106C CASCADE FOR (a) CLEAN INFLOW, AND (b) $3.2 \%$ INFLOW TURBULENCE. DIFFERENT PERSPECTIVE VIEWS ARE INCLUDED IN (i) AND (ii) FOR CLARITY.

sitive to the inflow turbulence except very close to the trailing edge region, but the peak wake loss drops by $34 \%$ in comparison to the clean inflow case. Similar observations have been reported by others [10]. For the T106C configuration with $R e=80,000$, a $3.2 \%$ inflow turbulence level changes the suction-side separation bubble topology and airfoil surface pressures, and results in a lower peak wake loss by $30 \%$. Similar qualitative change in bubble topology is also observed by [1]. The process of transition to turbulence is mainly due to the separated shear layer in both in T106A and T106C cases. However, for the T106C case the turbulent flow reattaches, causing the turbulence characteristics to begin shifting towards wall-bounded turbulence with non-zero inflow.

\section{ACKNOWLEDGMENT}

Funding for Anirban Garai was provided by the Advanced Air Transport Technology Project in the NASA Advanced Air Vehicles Program through the NASA Postdoctoral Program administrated by Oak Ridge Associated Universities (ORAU). 

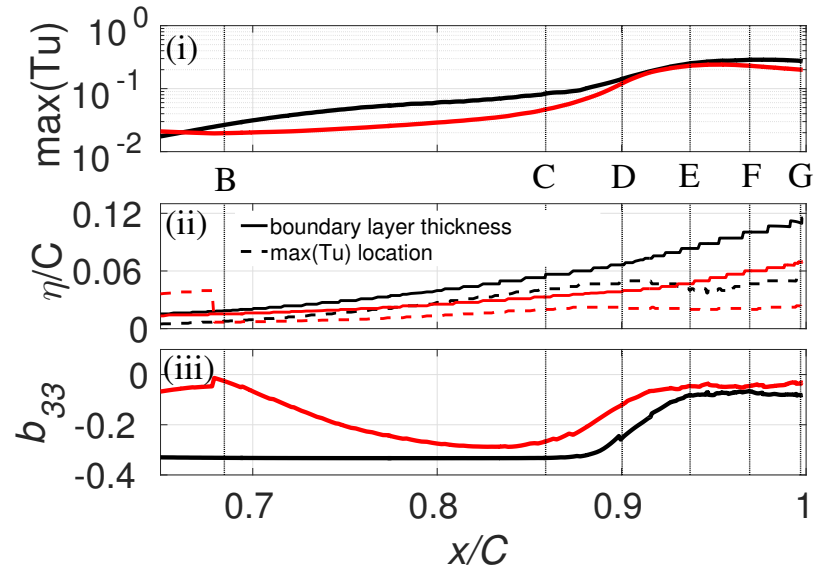

FIGURE 15: COMPARISON OF (i) Tu GROWTH RATE, (ii) BOUNDARY LAYER THICKNESS, MAXIMUM Tu LOCATION, AND (iii) $b_{33}$ FOR THE T106C CASCADE FOR CLEAN INFLOW (BLACK LINE) AND 3.2\% INFLOW TURBULENCE (RED LINE).

\section{REFERENCES}

[1] Michalek, J., Monaldi, M., and Arts, T., 2012. “Aerodynamic performance of a very high lift low pressure turbine airfoil (T106C) at low Reynolds and high Mach number with effect of free stream turbulence intensity". ASME Journal of Turbomachinery, 134, p. 061009.

[2] Mayle, R. E., 1991. "The role of laminar-turbulent transition in gas turbine engines". ASME Journal of Turbomachinery, 113, pp. 509-537.

[3] Wissink, J. G., 2003. "DNS of separating, low Reynolds number flow in a turbine cascade with incoming wakes.". International Journal of Heat and Fluid Flow, 24, pp. 626635.

[4] Rai, M. M., 2006. "A direct numerical simulation of transitional and turbulent flow on a turbine airfoil". AIAA Paper 2006-4460.

[5] Rai, M. M., 2009. "A direct numerical simulation of transition and turbulence in a turbine stage". AIAA Paper 2009584.

[6] Rai, M. M., 2010. "A direct numerical simulation of statorrotor interaction in an axial compressor". AIAA Paper 2010-6533.

[7] Rai, M. M., 2011. "A direct numerical simulation of flow through a low pressure turbine stage". AIAA Paper 20113092.

[8] Sandberg, R. D., Pichler, R., and Chen, L., 2012. "Assessing the sensitivity of turbine cascade flow to inflow disturbances using direct numerical simulation". 13th International Symposium for Unsteady Aerodynamics, Aeroacous-
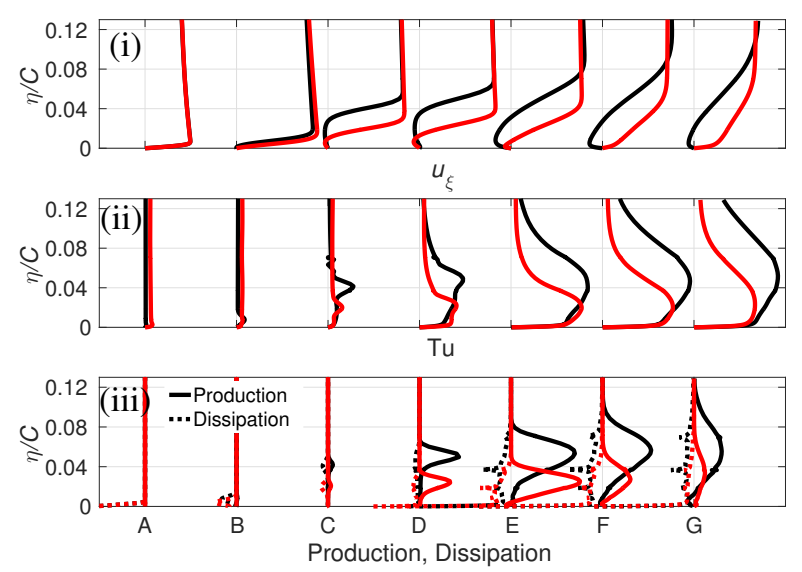

FIGURE 16: WALL NORMAL PROFILE OF (i) MEAN TANGENTIAL VELOCITY, (ii) TURBULENT INTENSITY, AND (iii) TURBULENT PRODUCTION AND DISSIPATION AT THE MONITORING STATIONS, AS SHOWN IN FIG. 14, ON THE SUCTION-SIDE OF THE T106C CASCADE FOR CLEAN INFLOW (BLACK COLORED LINE), AND 3.2\% INFLOW TURBULENCE (RED COLORED LINE).

tics and Aeroelsticity in Turbomachinery.

[9] Sandberg, R. D., Pichler, R., Chen, L., Johnstone, R., and Michelassi, V., 2014. "Compressible direct numerical simulation of low-pressure turbines: part I - methodology". ASME Turbo Expo Paper GT2014-25685.

[10] Michelassi, V., Chen, L., Pichler, R., and Sandberg, R. D., 2014. "Compressible direct numerical simulation of lowpressure turbines: part II - effect of inflow disturbances". ASME Turbo Expo Paper GT2014-25689.

[11] Bassi, F., Crivellini, A., Rebay, S., and Savini, M., 2005. "Discontinuous Galerkin solution of the Reynoldsaveraged Navier-Stokes and $k-\omega$ turbulence model equations". Computers and Fluids, 34, pp. 507-540.

[12] Corsini, A., Rispoli, F., and Santoriello, A., 2005. "A variational multiscale higher-order finite-element formulation for turbomachinery flow computations". Computer Methods in Applied Mechanics and Engineering, 194, pp. 47974823.

[13] Cherednichenko, S., Frey, C., and Ashcroft, G., 2012. “On the application of the Discontinuous Galerkin method to turbomachinery flows". European Congress on Computational Methods in Applied Sciences and Engineering.

[14] Ghidoni, A., Colombo, A., and Rebay, S., 2013. "Simulation of the transitional flow in a low pressure gas turbine cascade with a high-order Discontinuous Galerkin method". Journal of Fluids Engineering, 135, p. 071101.

[15] Carton de Wiart, C., Hillewaert, K., and Geuzaine, P., 2012. 
"DNS of a low pressure turbine blade computed with a Discontinuous Galerkin method". ASME Turbo Expo Paper GT2012-68900.

[16] Hillewaert, K., Carton de Wiart, C., Verheylewegen, G., and Arts, T., 2014. "Assessment of a high-order Discontinuous Galerkin method for the direct numerical simulation of transition at low-Reynolds number in the T106C highlift low pressure turbine cascade". ASME Turbo Expo Paper GT2014-26739.

[17] Carton de Wiart, C., Hillewaert, K., Lorriaux, E., and Verheylewegen, G., 2015. "Development of a Discontinuous Galerkin solver for high quality wall-resolved/modelled DNS and LES of practical turbomachinery flows on fully unstructured meshes". ASME Turbo Expo Paper GT201543428.

[18] Garai, A., Diosady, L. T., Murman, S. M., and Madavan, N. K., 2015. "DNS of flow in a low-pressure turbine cascade using a Discontinuous-Galerkin spectral-element method". ASME Turbo Expo Paper GT2015-42773.

[19] Eswaran, V., and Pope, S. B., 1988. "An examination of forcing in direct numerical simulations of turbulence". Computers and Fluids, 16, p. 257.

[20] Medic, G., and Sharma, O., 2012. "Large-eddy simulation of flow in a low-pressure turbine cascade". ASME Turbo Expo Paper GT2012-68878.

[21] Lundgren, T. S., 2003. "Linearly forced isotropic turbulence”. Annual Research Briefs, Center for Turbulence Research, pp. 461-473.

[22] Alvelius, K., 1999. "Random forcing of three-dimensional homogeneous turbulence". Physics of Fluids, 11, p. 1880.

[23] Ghosal, S., Lund, T., Moin, P., and Akselvoll, K., 1995. "A dynamic localization model for large-eddy simulations of turbulent flows". Journal of Fluid Mechanics, 286, p. 229.

[24] Ishihara, T., and Kaneda, Y., 2002. Statistical theories and computational approaches to turbulence. Springer, Berlin, ch. High resolution DNS of incompressible homogeneous forced turbulence - time dependence of the statistics.

[25] Sullivan, N., Mahalingam, S., and Kerr, R., 1994. "Determininstic forcing of homogeneous, isotropic turbulence". Physics of Fluids, 6, p. 1612.

[26] Wang, L., Chen, S., Brasseur, J., and Wyngaard, J., 1996. "Examination of hypotheses in the Kolmogorov refined turbulence theory through high-resolution simulations. part I - velocity fields". Journal of Fluid Mechanics, 309, p. 113.

[27] Carroll, P. L., and Blanquart, G., 2013. "A proposed modification to Lundgren's physical space velocity foricing method for isotropic turbulence". Physics of Fluids, 25, p. 105114.

[28] Rosales, C., and Meneveau, C., 2005. "Linear forcing in numerical simulations of isotropic turbulence: physical space implementation and convergence properties". Physics of Fluids, 17, p. 095106.
[29] Petersen, M. R., and Livescu, D., 2010. "Forcing for statistically compressible isotropic turbulence". Physics of Fluids, 22, p. 116101.

[30] Hughes, T. J. R., Franca, L. P., and Mallet, M., 1986. “A new finite element formulation for computational fluid dynamics I: symmetric forms of the compressible Euler and Navier-Stokes equations and the second law of thermodynamics". Computer Methods n Applied Mechanics and Engineering, 54(2), pp. 223-234.

[31] Chalot, F., Hughes, T. J. R., and Shakib, F., 1990. "Symmetrizaiton of conservative laws with entropy for hightemperature hypersonic computations". Computing Systems in Engineering, 1(2-4), pp. 495-521.

[32] Garai, A., Diosady, L. T., Murman, S. M., and Madavan, N. K., 2016. "Development of a perfectly matched layer technique for a Discontinuous-Galerkin spectral-element method". AIAA Paper 2016-1338.

[33] Diosady, L. T., and Murman, S. M., 2013. "Design of a variational multiscale method for turbulent compressible flows". AIAA Computational Fluid Dynamics Conference, 2013-2870.

[34] Diosady, L. T., and Murman, S. M., 2015. "Higher-order methods for compressible turbulent flows using entropy variables". AIAA Paper 2015-0294.

[35] Ismail, F., and Roe, P. L., 2009. "Affordable, entropyconsistent Euler flux functions II: entropy production at shocks". Journal of Computational Physics, 228, pp. 5410-5436.

[36] Bassi, F., and Rebay, S., 2000. "GMRES Discontinuous Galerkin solution of the compressible Navier-Stokes equations". In Discontinuous Galerkin Methods: Theory, Computation and Application, Springer, Berlin, pp. 197-208.

[37] Diosady, L. T., and Murman, S. M., 2014. "DNS of flows over periodic hills using a Discontinuous-Galerkin spectralelement method". AIAA Paper 2014-2784.

[38] Kida, S., and Orszag, S. A., 1990. "Energy and spectral dynamics in forced compressible turbulence". Journal of Scientific Computing, 5, pp. 85-125.

[39] Sarkar, S., 1992. "The pressure-dilatation correlation in compressible flows". Physics of Fluids A, 4, pp. 26742682.

[40] Blaisdell, G. A., Mansour, N. N., and Reynolds, W. C., 1993. "Compressibility effects on the growth and structure of homogeneous turbulent shear flow". Journal of Fluid Mechanics, 256, pp. 443-485.

[41] Stadtmuller, P., 2001. "Investigation of wake-induced transition on the LP turbine cascade T106A-EIZ". In DFGVerbundprojekt Fo 136//11, Version 1.0, University of the Armed Forces, Munich, Germany.

[42] Stieger, R. D., and Hodson, H. P., 2003. "The transition mechanism of highly-loaded LP turbine blades". ASME Turbo Expo Paper GT2003-38304. 